\title{
Richard Poulin
}

Sociologue, professeur titulaire, département de sociologie et d'anthropologie, Université d'Ottawa.

(2006)

\section{"Le système de prostitution militaire en Corée du Sud, en Thailande et aux Philippines."}

Un document produit en version numérique par Jean-Marie Tremblay, bénévole, professeur de sociologie retraité du Cégep de Chicoutimi

Courriel: jean-marie tremblay@uqac.ca

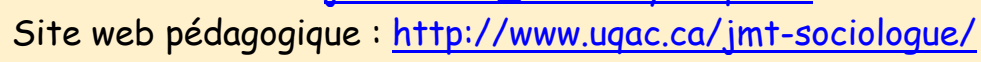

Dans le cadre de: "Les classiques des sciences sociales" Une bibliothèque numérique fondée et dirigée par Jean-Marie Tremblay, professeur de sociologie au Cégep de Chicoutimi

Site web: $\underline{h t t p: / / c l a s s i q u e s . u q a c . c a l}$

Une collection développée en collaboration avec la Bibliothèque

Paul-Émile-Boulet de l'Université du Québec à Chicoutimi

Site web: $h+t p: / /$ bibliotheque.uqac.cal 


\section{Politique d'utilisation de la bibliothèque des Classiques}

Toute reproduction et rediffusion de nos fichiers est interdite, même avec la mention de leur provenance, sans l'autorisation formelle, écrite, du fondateur des Classiques des sciences sociales, Jean-Marie Tremblay, sociologue.

Les fichiers des Classiques des sciences sociales ne peuvent sans autorisation formelle:

- être hébergés (en fichier ou page web, en totalité ou en partie) sur un serveur autre que celui des Classiques.

- servir de base de travail à un autre fichier modifié ensuite par tout autre moyen (couleur, police, mise en page, extraits, support, etc...),

Les fichiers (.html, .doc, .pdf, .rtf, .jpg, .gif) disponibles sur le site Les Classiques des sciences sociales sont la propriété des Classiques des sciences sociales, un organisme à but non lucratif composé exclusivement de bénévoles.

Ils sont disponibles pour une utilisation intellectuelle et personnelle et, en aucun cas, commerciale. Toute utilisation à des fins commerciales des fichiers sur ce site est strictement interdite et toute rediffusion est également strictement interdite.

L'accès à notre travail est libre et gratuit à tous les utilisateurs. C'est notre mission.

Jean-Marie Tremblay, sociologue

Fondateur et Président-directeur général, LES CLASSIQUES DES SCIENCES SOCIALES. 
Cette édition électronique a été réalisée par Jean-Marie Tremblay, bénévole, professeur de sociologie au Cégep de Chicoutimi à partir de:

\section{Richard POULIN}

"Le système de prostitution militaire en Corée du Sud, en Thaïlande et aux Philippines".

Un article publié dans la revue Bulletin d'histoire politique, vol. 15 , no 1, automne 2006, pp. 81-92. Un numéro intitulé : "Sexualité et politique".

[Autorisation formelle accordée par l'auteur le 13 septembre 2011 de diffuser ce texte dans Les Classiques des sciences sociales.]

Courriel : Richard.Poulin@uottawa.ca

Polices de caractères utilisée : Comic Sans, 12 points.

Édition électronique réalisée avec le traitement de textes Microsoft Word 2008 pour Macintosh.

Mise en page sur papier format : LETTRE US, $8.5^{\prime \prime} \times 11^{\prime \prime}$.

Édition numérique réalisée le 4 septembre 2011 à Chicoutimi, Ville de Saguenay, Royaume du Saguenay, Québec. 


\section{Richard Poulin}

Sociologue, professeur titulaire, département de sociologie et d'anthropologie, Université d'Ottawa.

\section{"Le système de prostitution militaire en Corée du Sud, en Thaillande et aux Philippines."}

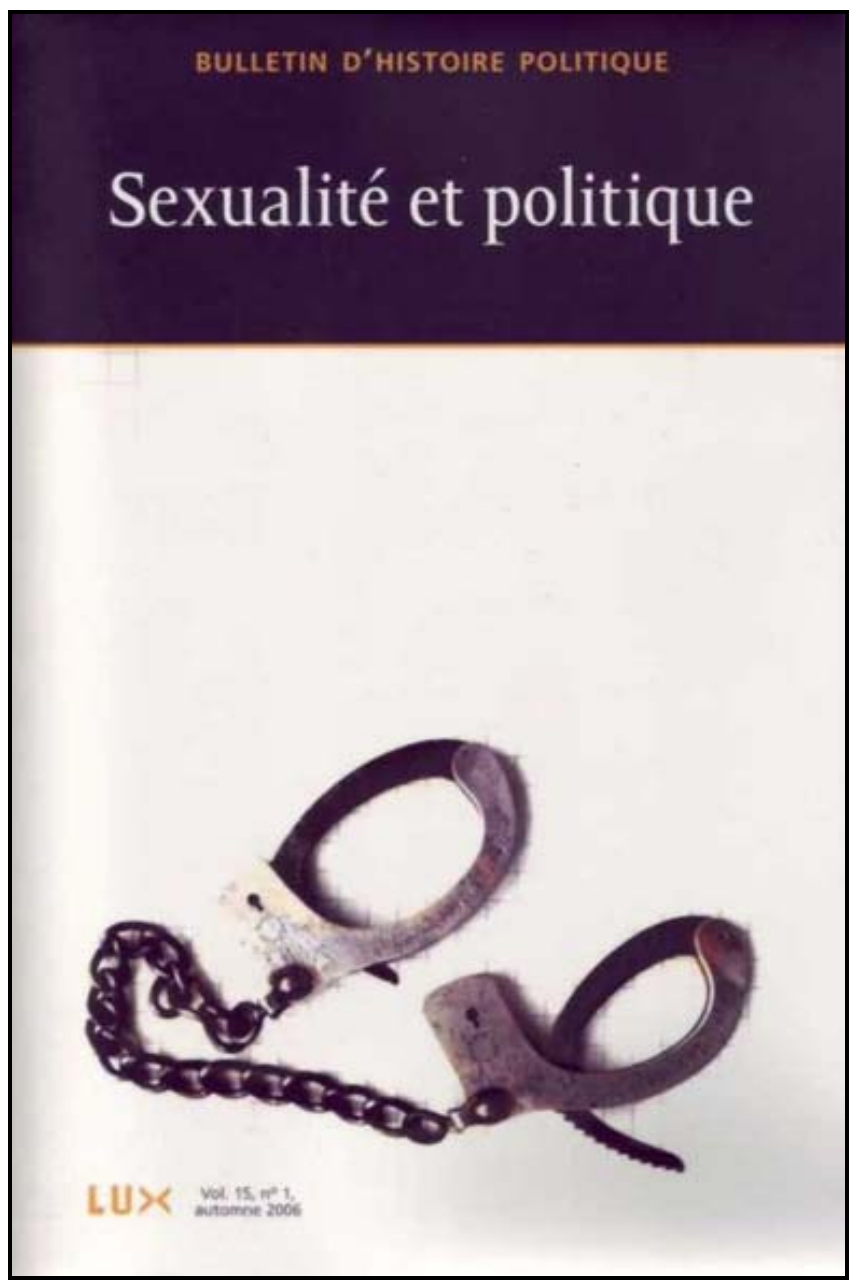

Un article publié dans la revue Bulletin d'histoire politique, vol. 15, no 1, automne 2006, pp. 81-92. Un numéro intitulé : "Sexualité et politique". 


\section{Table des matières}

Introduction

Les bordels de « réconfort » japonais

Les installations « récréatives » de Corée

Les rest and recreation sites en Thailande et aux Philippines

Forces de maintien de la paix et prostitution

Conclusion 
Richard Poulin

Sociologue, professeur titulaire, département de sociologie et d'anthropologie, Université d'Ottawa.

"Le système de prostitution militaire en Corée du Sud, en Thailande et aux Philippines".

Un article publié dans la revue Bulletin d'histoire politique, vol. 15, no 1, automne 2006, pp. 81-92. Un numéro intitulé : "Sexualité et politique".

\section{Introduction}

\section{Retour à la table des matières}

Les invasions et les occupations militaires ont été un facteur décisif dans la mondialisation des industries du sexe ${ }^{1}$. Les guerres modernes ont engendré un développement considérable de la prostitution et impliqué son organisation «rationnelle» au profit des armées. Des centaines de milliers si ce n'est des millions de femmes et de fillettes ont été mises au service sexuel des militaires dans des maisons closes financées et organisées par les États. Les exemples sont nombreux: comfort women pour les militaires japonais, aux camptowns et rest and recreation sites pour les GI en passant par les BMC (bordels militaires de campagne) pour les troupes françaises et coloniales 2 et les « établissements sanitaires » allemands pendant la Deuxième Guerre mondiale ${ }^{3}$. Évidemment, l'aménagement rationnel de la prostitution pour

1 J'ai traité plus en profondeur de ces questions dans mon livre, La mondialisation des industries du sexe. Prostitution, pornographie, traite des femmes et des enfants, Ottawa, L'Interligne, 2004.

2 La documentation sur cette question est relativement pauvre, mais récemment étaient publiés deux livres d'inégale valeur: Michel Serge Hardy, De la morale au moral des troupes ou l'histoire des BMC, 1918-2004, Panazole, Lavauzelle, 2004 et Christelle Tarraud, La prostitution coloniale en Algérie, en Tunisie et au Maroc de 1830 à 1962, Paris Payot, 2003.

3 La Wehrmacht a effectué des rafles dans toute l'Europe pour peupler les « bordels», dits « établissements sanitaires», au profit de ses troupes. Il en existait 
les armées exigeait, entre autres, l'organisation de la traite des femmes et des enfants à des fins de prostitution.

Je ne pourrai examiner dans le cadre de cet article l'ensemble des cas. Je concentrerai mon analyse sur les occupations militaires en Asie. D'une part, l'industrie de masse de la prostitution y a connu un essor à la faveur des guerres de Corée et du Vietnam et du stationnement des troupes aux Philippines et en Thaillande, bases arrière dans la lutte contre les communistes coréens et vietnamiens. D'autre part, la croissance importante de la prostitution a permis l'établissement de l'infrastructure nécessaire au développement du tourisme sexuel d'aujourd'hui. Au cours de la seule décennie quatre-vingt-dix, plus de 33 millions de femmes et d'enfants de l'Asie du Sud-Est auraient été victimes de la traite à des fins de prostitution 4 .

\section{Les bordels de " réconfort » japonais}

\section{Retour à la table des matières}

Entre 1932 et 1945, le Japon impérial a fait la guerre à plusieurs pays asiatiques - dont nombre d'entre eux étaient des colonies de la Grande-Bretagne, de la France ou des Pays-Bas - et aux États-Unis. Cette guerre a vu naître les bordels militaires de "réconfort» ou « centres de délassement 5 ». Au début de 1932, les troupes nippones ont établi à Shanghai le premier bordel de réconfort. Le système a été institutionnalisé en 1937-1938 lorsque le Japon a entrepris la conquête

pour les officiers, pour les soldats, pour les travailleurs des chantiers de l'organisation Todt et dans des camps de concentration. Voir Christa Paul, Zwangsprostitution : staatliche Bordelle im Nationalsozialismus, Berlin, Hentrich Verlag, 1994, et Insa Meinen, Wehrmacht und Prostitution im besetzten Frankreich, Bremen, Temmen Verlag, 2002.

4 Jenna Shearer Demir, Trafficking of Women for Sexual Exploitation: A Gender-based Well-founded Fear? An Examination of Refugee Status Determination for Trafficked Prostituted Women from CEE/CIS Countries to Western Europe [en ligne], 2003 [site visité le 10 octobre 2003], http://www.antislavery.org/homepage/traffic\%20news/shearerdemir 2004.pdf.

5 George L. Hicks, Comfort Women : Japan's Brutal Regime of Enforced Prostitution in the Second World War, New York, W.W. Norton \& Co, 1994. 
de la Chine ${ }^{6}$. Au début des années quarante, les bordels militaires de réconfort ont été mis en place dans l'ensemble des territoires occupés (Indonésie, Indochine, Thaillande, Birmanie, Bornéo, Philippines, etc.) et dans l'empire lui-même (Japon, Okinawa) ainsi que dans ses «vieilles» colonies (Corée et Taiwan). Les «centres de délassement» étaient situés principalement près du front - là où il y avait le plus de troupes -, mais aussi au Japon même où, malgré la prostitution légale, des bordels de « réconfort» ont été créés à l'intention de ceux qui n'avaient pas les moyens de se payer les lupanars « classiques» prostituant des Japonaises.

Le nombre exact de femmes prostituées dans les bordels militaires japonais est inconnu parce que le gouvernement a détruit les archives les concernant 7 . Sur la base de témoignages 8 et de quelques documents, qui indiquent la proportion de femmes de « réconfort»par rapport au nombre de soldats japonais, les historiens estiment entre 80000 et 280000 - plus vraisemblablement 2000009 - les prostituées au service des militaires. Les femmes de « réconfort», ianfu en japonais, étaient très largement des Asiatiques de différentes nationalités (Japonaises, Taiwanaises, Indonésiennes ou Philippines), mais à $80 \%$ Coréennes. Quelques-unes étaient des Néerlandaises capturées en Indonésie 10.

6 Radhika Coomaraswarmy, Report on the Mission to the Democratic People's Republic of Korea, the Republic of Korea and Japan on the Issues of Military Sexual Slavery in Wartime, U.N. Commission on Human Rights, $52^{\text {nd }}$ Sess., Prov. Agenda Item 9(a), at 2, U.N. Doc. E/CN.4/1996/53/Add.1, 1996.

7 Pyong Gap Min, «Korean "comfort women": The intersection of colonial power, gender, and class », Gender \& Society, vol. 17, n 6, December, 2003, p. 941.

8 Quelque 450 femmes de « réconfort» survivantes ont été identifiées en Corée du Sud, en Corée du Nord, en Chine et dans d'autres pays asiatiques, 60 à Taiwan (Min, op. cit, p. 954) et deux aux Pays-Bas.

9 Voir Gay McDougal, Systematic Rape, Sexual Slavery and Slavery-like Practices during Armed Conflict: Update to the Final Report, U.N. Economic and Social Council, 52 ${ }^{\text {nd }}$ Sess., Agenda Item 6(a), UN Doc. E/CN.4/Sub.2/21, 2000, et Yoshiaki Yoshimi, "Comfort Women": Sexual Slavery in the Japanese Military During WWII, Columbia University Press, 2000.

10 Hicks, op. cit., p. 58-59. 
L'esclavage sexuel au profit de l'armée japonaise était, en partie, un sous-produit de la colonisation par le Japon de la Corée ${ }^{11}$. La Corée est devenue un protectorat du Japon en 1905 et a été annexée en 1910. Le gouvernement japonais a également recruté des Taiwanaises, mais si le nombre de «femmes de réconfort» de cette ancienne colonie est moins important que celui de Corée, c'est essentiellement parce que Taiwan était moins peuplé 12 .

Les femmes prostituées pour le bénéfice de la troupe étaient considérées comme inférieures d'un point de vue racial 13 , ce qui légitimait leur esclavage sexuel. Les Japonaises de « réconfort » étaient réservées aux officiers tandis que les femmes des autres nationalités étaient prostituées au profit des militaires du rang 14 et étaient traitées plus brutalement que les Japonaises ${ }^{15}$. Les vierges étaient particulièrement prisées et recherchées, sauf pour les Nippones de « réconfort » qui, lors de leur recrutement, étaient déjà des « professionnelles » du secteur de « divertissement » pour hommes au Japon même ${ }^{16}$. Elles étaient plus âgées en moyenne que les Coréennes. Presque toutes les Coréennes étaient vierges, célibataires et jeunes, âgées de 11 ans au début de la vingtaine 17.

11 Ce qui a affecté d'autres catégories de population. En août 1945, à la fin de la guerre, il y avait plus de 200000 soldats coréens dans l'armée japonaise. Plus de 150000 civils coréens ont dû travailler comme paramilitaires dans des zones de guerre. Entre 1939 et 1945, 668000 manœuvres coréens ont été envoyés au Japon (Min, op. cit., p 943-944).

12 La population coréenne était estimée en 1944 à 25 millions de personnes comparativement à 4 millions à Taiwan.

13 Ustinia Dolgopol et Snehal Paranjape, Comfort Women: An Unfinished Ordeal, Report of a Mission, Geneva, International Commission of Jurists, 1994, p. 42.

14 Yoshiyuki Tanaka, Japan's Comfort Women. Sexual Slaverty and Prostitution during World War II and US Occupation, New York, Roultedge, 2002, p. 34.

15 C. Yun, « Women's "volunteer corps" », Women and Tourism, South Korea, Korea Church Women United, 1988, p. 67.

16 Tanaka, op. cit., p. 41.

17 Lisa Go, An Unbroken History of Japan's Sex Slaves, [en ligne], Newsletter, The International Communication Project, April 1994 [site consulté le 20 novembre 2003], 
Les conditions de vie des femmes de « réconfort » étaient extrêmement dures. Elles pouvaient recevoir jusqu'à 70 hommes par jour. Souvent battues, torturées ou mutilées, certaines ont été exécutées par des soldats insatisfaits. L'armée avait fixé les règles de fonctionnement, notamment les horaires (de neuf heures du matin à minuit, tous les jours de la semaine), les jours de repos (deux jours par mois lors des menstrues), les désinfections des chambres-cellules, les visites médicales ou encore le traitement des infections vénériennes.

Selon les époques, les bordels de « réconfort » étaient dirigés soit directement par l'armée, soit par les gouvernements locaux ou encore par des particuliers. Au fur et à mesure de la propagation des centres, l'armée a peu à peu abandonné ses prérogatives de fonctionnement et de gestion aux civils ${ }^{18}$. L'armée leur a accordé un statut paramilitaire et assurait les services de transport et, de façon croissante, le recrutement. Elle a conservé un droit de regard, en matière de santé notamment ${ }^{19}$. Les tenanciers proxénètes touchaient un pourcentage sur le prix des passes, bien souvent les femmes ne recevaient rien.

Selon les témoignages des survivantes 20 , trois méthodes de recrutement ont été établies : le racolage de «volontaires » qui étaient déjà prostituées; la duperie sous forme d'offres fallacieuses d'emplois bien rémunérés de serveuses, de cuisinières ou de lingères pour l'armée et, enfin, la coercition et les razzias.

http://www.csf.colorado.edu/ipe/Geographic_Archives/asia/women/Japanese_ Sex_Slaves.txt.

18 Cette industrialisation de la prostitution et de la traite à des fins de prostitution a laissé des traces, notamment dans le vocabulaire. Encore aujourd'hui, tant à Taiwan qu'au Cambodge, c'est par une expression japonaise, mama-san, que l'on nomme les mères-maquerelles, c'est-à-dire les tenancières de bordel. Voir, entre autres, Somaly Mam, Le silence de l'innocence, Paris, Anne-Carrière, 2005.

19 Les camps de «femmes de réconfort» étaient strictement surveillés sur la base d'instructions émanant du ministère des Armées, notamment en matière de santé, pour éviter la propagation des infections vénériennes. Voir à ce sujet, Yoshimi, op. cit. p. 128.

20 Voir notamment, Dai Sil Kim-Gibson, Silence Broken: Korean Comfort Women, Parkersbourg, Mid-Prairie Book, 1999 et Sangmie Choi Schellstede, dir., Comfort Women Speak: Testimony of Sex Slaves of the Japanese Military, New York, Holmes \& Meier, 2000. 
Des cadets officiers nippons ont reçu une formation spéciale de l'armée pour apprendre à bien gérer l'approvisionnement en marchandises inanimées et vivantes pour le « réconfort» des soldats ${ }^{21}$. Les corps expéditionnaires envoyaient en Corée des marchands chargés de recruter des Coréennes destinées à devenir des esclaves sexuelles de la troupe, de connivence ou avec l'aide de la police militaire ou de la police locale. Ces agents étaient généralement désignés par l'étatmajor mais aussi parfois directement par les divisions, les brigades ou les régiments 22 .

Le programme des « centres de délassement » a donc été méthodique, soigneusement organisé et exécuté par le gouvernement japonais.

Quelques jours seulement après la défaite japonaise, l'Association pour la création de facilités récréatives spéciales, financée indirectement par le gouvernement japonais, ouvrait, à Tokyo, un premier bordel de réconfort pour les troupes alliées d'occupation. $\grave{A}$ son point culminant, cette association exploitait la prostitution de 70000 femmes. L'armée américaine n'a pas simplement utilisé les maisons closes mises à sa disposition, elle a aussi réclamé la construction d'autres bordels 23 qu'elle a gérés conjointement avec les autorités japonaises. Cette politique a perduré. Ainsi, pendant la guerre du Vietnam, Okinawa a servi de rest and recreation site pour les troupes états-uniennes. La majorité des prostituées desservant la base militaire de Kadena à Okinawa était originaire des Philippines et de la Thaïlande ${ }^{24}$, victimes de la traite à des fins de prostitution.

21 Tanaka, op. cit.

22 Coomaraswamy, op. cit.

23 Yoshimi, op. cit., p. 233.

24 Katherine Moon, Sex Among Allies: Military Prostitution in U.S./Korea Relations, Irvington, NY, Columbia University Press, 1997, p. 48. 


\section{Les installations « récréatives » de Corée}

\section{Retour à la table des matières}

En 1954, les gouvernements des États-Unis et de la République de Corée ont signé un traité de défense mutuelle qui a formellement accordé des bases militaires aux troupes états-uniennes en Corée du Sud. Une des clauses du traité prévoyait la mise en place de rest and recreation sites, ou kijichion, pour les soldats américains 25 . Dans ces centres, les bordels étaient subventionnés par le gouvernement coréen qui a pu ainsi édicter ses règles: les prostituées devaient « desservir» 29 soldats par jour. Le gouvernement a même évalué que les contacts sexuels ne devaient pas excéder 30 minutes. Les personnes prostituées pour les militaires de ces bordels devaient s'enregistrer auprès du gouvernement coréen. L'uniforme militaire accordait automatiquement un droit d'entrée au soldat, sans aucune autre formalité ni contrôle 26 . Dans les années soixante, le prix de la passe était 2 \$ ou moins l'heure et 5 ou 10 \$ la nuit. Dans les années quatre-vingt, la nuit coûtait 20 \$ et l'heure 10 \$. Les propriétaires des bordels et les proxénètes récupéraient, en moyenne, $80 \%$ de l'argent et les femmes prostituées $20 \% 27$.

Les camptowns ont été établis conjointement par les autorités coréennes et états-uniennes, sous les auspices du Conseil de la police métropolitaine et des propriétaires des industries du sexe, financièrement indemnisés par le gouvernement japonais ${ }^{28}$. Avant la guerre de Corée, entre 1945 et 1949, la prostitution pour les troupes d'occupation états-uniennes était inorganisée et non règlementée. La

25 Jennifer Latstetter, American Military-Base Prostitution [en ligne], Williamsburg, The College of William \& Mary, 2000 [site visité le 5 mai 2003], http://www.wm.edu/SO/monitor/spring2000/ paper6.htm.

26 Moon, op. cit, p. 167.

27 Sandra Strudevant et Brenda Stolzfus, Let the Good Times Roll. Prostitution and the U.S. Military in Asia, New York, The New Press, 1992, p. 192.

28 Mire Koikari, «Rethinking gender and power in the U.S. occupation of Japan, 1945-1952 », Gender \& History, vol. 11, n², 1999, p. 321. 
guerre de Corée a créé les conditions sociales et économiques, notamment la disponibilité des «matières premières» 29 , pour la mise en place du système des kijich'on. La guerre et la pauvreté qui l'accompagne, le chaos social et politique, la séparation de familles et les millions de jeunes orphelines et de veuves, sans toit ni gagne-pain, ont permis la «fabrication en série» des prostituées facilement recrutées par le gouvernement sur promesse d'un emploi gouvernemental bien payé qui s'avérait, en fait, celui de prostituée pour les GI 30.

Au milieu des années quatre-vingt-dix, on dénombrait 18000 personnes prostituées enregistrées et 9000 non enregistrées au service des 43000 militaires états-uniens stationnés en Corée ${ }^{31}$. En 2000, 8500 femmes originaires des Philippines et de Russie ont été victimes de la traite aux fins de prostitution pour les militaires américains de Corée 32 . Elles ont pu entrer au pays au moyen de visas de « divertissement » délivrés par le gouvernement à la suite de négociations avec l'Association des propriétaires de bars. Des 41 principaux camps militaires américains en Corée, les 12 plus grands bénéficient de la présence du voisinage de camptowns. Les civils coréens y sont interdits d'entrée. En 2000, environ deux millions de prostitueurs 33 ont fait un séjour dans les camptowns.

Comme l'industrie de la prostitution permettait des revenus importants au gouvernement coréen, ce dernier a adopté des politiques de «développement économique» responsables de la croissance de l'industrie du sexe. À partir de l'automne de 1973, il a entrepris une

29 Moon, op. cit., p. 35.

30 Rita Nakashima Brock et Susan Brooks Thistlethwaite, Casting Stones. Prostitution and Liberation in Asia and the United States, Minneapolis, Fortress Press, 1996, p. 73.

31 Latstetter, op. cit.

32 Donald Macintyre, «Base Instincts», Time Asia, vol. 160, n 5, August 12, 2000.

33 J'emploie le terme «prostitueur» (le terme «prostituant» pouvant y être substitué) pour désigner le « client» de la prostitution, refusant de considérer la prostitution sous le seul angle d'une «opération commerciale» entre un «acheteur » et une «vendeuse», ce qui m'apparaît être une réduction libérale négationniste d'un système d'asservissement et où le proxénète est singulièrement oublié. Des termes équivalents ont été développés dans de nombreuses langues. 
politique d'expansion du tourisme basé sur la marchandisation du corps des femmes ${ }^{34}$, le tourisme kisaeng. Le nombre de touristes japonais - à $85 \%$ des hommes seuls - a grimpé en flèche : de 96531 en 1971 à 649707 en 1979. En 1978, les revenus de la prostitution sont estimés à 700 milliards de won ${ }^{35}$. En 1989, l'industrie du « divertissement » pour hommes représentait $5 \%$ du PNB du pays et prostituait entre 1,2 et 1,5 million de Coréennes (soit le cinquième de la cohorte des femmes âgées entre 15 et 29 ans) pour 2,34 millions de touristes étrangers en 1988, l'année des Jeux olympiques de Séoul.

Jusqu'au début des années quatre-vingt-dix, les prostituées étaient essentiellement d'origine coréenne, mais avec l'expansion des industries du sexe, la Corée est devenue un pays de destination de la traite des femmes.

\section{Les rest and recreation sites en Thailande et aux Philippines}

\section{Retour à la table des matières}

À la différence de ceux de la Corée, les rest and recreation sites déployés pendant la guerre du Vietnam n'ont pas tous été directement rattachés aux bases militaires 36 . Plusieurs établissements étaient situés en Thaillande et aux Philippines, une ancienne colonie américaine. Les États-Unis ont conclu une entente avec la Thaillande en 1967 pour que le pays soit un lieu « de repos et de loisir» pour ses soldats. L'effet évident de cette entente a été que le corps des femmes de Thailande a servi de moyen d'accumulation de capital pour les autorités

34 Selon Moon, op. cit., p. 179, l'Association coréenne de tourisme international (KITA) a créé un Certificat d'emploi dans le secteur de divertissement qui était, en fait, un permis pour la prostitution et conférait le droit d'effectuer des passes dans des hôtels.

35 Moon, op. cit., p. 142.

36 Lors du retrait des troupes états-uniennes du Vietnam, il y avait environ 500000 femmes en situation de prostitution dans le pays. Kathleen Barry, The Prostitution of Sexuality, New York \& London, New York University Press, 1995, p. 135. 
du pays. Ainsi, c'est un général de la Royal Air Force thaillandaise qui a négocié l'accord qui a permis un afflux énorme de devises fortes dans l'économie du pays. Son épouse a dirigé la première agence de tours sexuels de la Thaillande pour les militaires américains 37 . Approximativement 4 millions de dollars américains ont été prêtés, entre autres, par la Chase Manhattan Bank, pour financer la construction des rest and recreation sites. Entre 1962 et 1975, environ 70000 militaires américains ont pu, chaque année, "se reposer et reprendre des forces $\gg$ dans les bordels thaillandais.

L'essor de la prostitution en Thaillande a véritablement débuté avec la guerre du Vietnam. En 1957, il y avait 20000 personnes prostituées: en 1964, après l'établissement de sept bases états-uniennes dans le pays, le nombre de prostituées a atteint le chiffre de 400000. Pendant la guerre du Vietnam, la Banque mondiale a recommandé à la Thailande d'adopter comme stratégie économique le développement du tourisme 38 . Depuis, la prostitution y est une industrie de masse : en 1993, le nombre de personnes prostituées était estimé à deux millions, le tiers étant des mineur-es. En 1995, les revenus de la prostitution en Thailande représentaient près de 59 à $60 \%$ du budget du gouvernement. En 1998, l'Organisation internationale du travail estimait que la prostitution constituait $14 \%$ de l'ensemble des activités économiques du pays 39.

La prostitution autour des bases militaires états-uniennes a également joué un rôle significatif aux Philippines. Jusqu'au départ des troupes, en 1992, était organisé dans ce pays l'un des plus grands centres « de repos et de loisir»d'Asie. À Olongapo et à Angeles City, deux bases américaines près de Manille, le problème des infections vénériennes était si important que le gouvernement américain a financé l'établissement de cliniques médicales. Ces cliniques, cependant, n'étaient accessibles qu'aux 6000 « hôtesses» licenciées pour « des-

37 Brock et Brooks Thistlehwaite, op. cit., p 116.

38 Lillian S. Robinson, « Touring Thailand's sex industry », The Nation, November 1 , 1993, p. 496.

39 Sheila Jeffreys, «Globalizing sexual exploitation: Sex tourism and the traffic in women », Leisure Studies, vol. 18, n 3, july 1999, p. 185. 
servir » les GI, pas aux prostituées non enregistrées 40 . Il n'y avait pratiquement aucune industrie à Olongapo et Angeles sauf celle de « divertissement » pour les GI, avec approximativement 55000 prostituées enregistrées et non enregistrées et un total de 2182 rest and recreation sites ${ }^{41}$. Au départ des troupes, quelque 50000 enfants américano-philippins ont été abandonnés par leurs pères. Au début des années quatre-vingt-dix, le nombre de femmes prostituées était estimé de 300000 à 500000 42. En 1999, quand les forces américaines sont retournées aux Philippines, le nombre d'« artistes» inscrites à Angeles City a presque doublé.

L'utilisation d'installations récréatives du type rest and recreation fait encore partie des politiques du Pentagone. Immédiatement après la première guerre contre l'Irak, les troupes états-uniennes victorieuses ont été envoyées en Thaillande pour y prendre du « bon temps » 43 .

\section{Forces de maintien de la paix et prostitution}

\section{Retour à la table des matières}

La présence de la force de maintien de la paix des Nations Unies et la libéralisation économique des pays sont des facteurs décisifs dans la prolifération des infrastructures de prostitution. Un exemple parmi d'autres: au Cambodge, en 1990, à l'époque du gouvernement provietnamien, on estimait le nombre de femmes prostituées à 1500 . L'arrivée des Casques bleus et des administrateurs civils de Mission préparatoire des Nations Unies au Cambodge en 1991, remplacée I'année suivante par l'Autorité transitoire des Nations Unies au Cambodge (Apronuc), a créé une explosion de la demande de jeunes femmes et de fillettes dans la prostitution. Durant cette période, beaucoup de jeu-

40 Yayori Matsui, «Sexual exploitation of women: Child prostitution and the expanding sex industry », Women's Asia, New York, Zed Books, 1989, p. 72.

41 Moon, op. cit., p. 155.

42 Barry, op. cit., p. 148.

43 Meredeth Turshen and Briavel Holcomb, Women's Lives and Public Policy: The International Experience, Westport, Praeger Publishers, 1993, p. 135. 
nes femmes et de fillettes de la campagne étaient victimes de la traite intérieure à des fins de prostitution vers les villes du pays pour satisfaire la demande des prostitueurs. En 1993, lors du retrait de I'Apronuc, 20000 personnes, principalement des femmes et des fillettes, étaient prostituées 44 . Avec la libéralisation économique et la transition au capitalisme, le Cambodge a connu une nouvelle explosion de l'industrie de la prostitution: en 1996, on évaluait à 57000 le nombre de jeunes femmes et de fillettes prostituées. Le Cambodge est désormais une destination prisée des prostitueurs internationaux (touristes sexuels), plus particulièrement des pédoprostitueurs, ainsi qu'un pays de transit et de destination des femmes et enfants victimes de la traite à des fins de prostitution. L'Organisation mondiale de la santé a tiré la sonnette d'alarme: dans les années à venir l'épidémie du sida risque de faire plus de victimes au Cambodge que le génocide orchestré par les Khmers rouges entre 1975 et 1979.

Le cas cambodgien n'est pas unique. La prostitution des femmes a également augmenté de façon dramatique avec la présence de la mission de pacification en Bosnie-Herzégovine. La mise en place de l'Arizona Market en Bosnie est, à cet égard, édifiante. Ce vaste marché détaxé, créé en 1992 par la SFOR (Force de stabilisation de I'OTAN), porte le nom d'un désert américain, car les autorités militaires des États-Unis y ont initié la création d'une zone franche «pour réconcilier par le commerce» les populations serbo-croate et bosniaque. Dans cette zone du Nord du pays, laissée sous autorité américaine et internationale après 1999 , le système proxénète a établi son marché. Les femmes y sont vendues comme l'étaient les esclaves victimes de la traite des négriers. L'Organisation internationale pour les migrations évalue à 10000 le nombre de personnes prostituées clandestines en Bosnie. Elle estime, en outre, que 250000 femmes et enfants de l'Europe de l'Est sont victimes de la traite via la Serbie et les États voisins, dont un grand nombre se retrouve dans les nouveaux protectorats internationaux de Bosnie et du Kosovo pour desservir soldats de I'OTAN, policiers de I'ONU et membres des ONG.

44 Somaly Mam et Emmanuel Dialma, « Apparences de droit et réalités de la traite humaine en Asie», dans Poulin, Richard (dir.), Prostitution, la mondialisation incarnée, Alternatives Sud, vol. XII, n 3, 2005, p. 89-109. 


\section{Conclusion}

Retour à la table des matières

À première vue, il peut sembler paradoxal que, dans un pays qui a connu les horreurs de guerre civile, certaines des violations des droits humains les plus élémentaires soient commises par la communauté internationale censée apporter la paix et permettre la reconstruction du pays. Toutefois, ce paradoxe n'en est pas un: le stationnement de troupes armées développe inévitablement les infrastructures prostitutionnelles et, par conséquent, la traite des femmes et des enfants aux fins de prostitution. Cet essor se traduit également par une augmentation de la clientèle locale et régionale. La mise en place de telles infrastructures est encouragée sinon pilotée par les forces d'occupation, y compris celles censées « maintenir la paix 》.

L'un des effets des occupations militaires en Asie a été le développement rapide et colossal des industries du sexe et la prostitutionnalisation du tissu social au point tel que de nombreux analystes considèrent que la prostitution fait partie intégrante de la culture de ces pays. Il est vrai que les infrastructures prostitutionnelles de masse ont généré une banalisation du sexe vénal, ce qui a permis une expansion importante de la « clientèle » locale ${ }^{45}$. Elles ont aussi pour conséquence l'essor de la traite des femmes et des enfants à des fins de prostitution pour fournir les industries du sexe dont la demande est pratiquement infinie. La croissance dramatique de la prostitution locale a permis l'établissement de l'infrastructure nécessaire au développement du tourisme sexuel, grâce notamment à la disponibilité de la «main-d'œuvre-marchandise » générée par la présence militaire. Des loisirs plus importants, des facilités de communications et de déplacement vers l'étranger, la construction sociale d'une image exotique et sensuelle des personnes prostituées asiatiques, qui seraient, grâce à leur culture, sexuellement matures malgré leur jeune âge, et les politi-

45 Pour 5,4 millions de touristes sexuels par an en Thaillande, on compte désormais 450000 prostitueurs locaux par jour (Jeffreys, op. cit., p. 186-187). Aujourd'hui, $75 \%$ des Thaillandais sont des prostitueurs occasionnels ou réguliers. 
ques gouvernementales favorables au tourisme sexuel ont contribué à l'explosion de cette industrie.

S'il y a « une culture de prostitution » en Corée du Sud, en Thailande et aux Philippines, elle est le résultat de politiques de « développement économique » au détriment des femmes et des enfants de ces pays. Cette stratégie a été préconisée par des organisations internationales comme la Banque mondiale et le Fonds monétaire international, qui ont accordé des prêts importants pour le développement des industries touristiques et de divertissement, source de devises fortes. Dans chacun des cas, ces politiques ont engendré une forte progression des industries du sexe.

Les interventions armées étrangères dans ces sociétés ont contribué de façon décisive à l'histoire de la prostitution contemporaine. Le cas coréen est significatif à cet égard. En 1916, le gouvernement japonais a légalisé la prostitution pour la première fois dans l'histoire coréenne et a importé des prostituées japonaises pour l'élite nippone de sa colonie 46. Rapidement, la prostitution locale s'est développée et des red lights districts ont vu le jour. L'organisation de la prostitution a atteint son apogée pendant la Deuxième Guerre mondiale avec les femmes et fillettes de "réconfort». Les « centres de délassement» de l'armée japonaise ont été en quelque sorte le prototype historique des rest and recreation sites au profit des troupes d'occupation étatsuniennes. Des femmes de "réconfort » pour les militaires japonais ont même fait partie de la première génération des prostituées des kijich'on 47.

La thèse du relativisme culturel camoufle les enjeux. La pratique des viols massifs en temps de guerre 48 et du développement « centres de loisir» pour les soldats - qui constituent l'avers et le revers d'une même médaille - ne sont pas l'apanage d'une culture ou d'une na-

46 Young Mi Pak, « U.S. Military Presence in Korea and Its Effect on Women», p. 23, cité par Moon, Katherine, op. cit., p. 187.

47 Latstetter, op. cit.

48 Voir à ce sujet l'essai de Bolya, La profanation des vagins. Le viol, arme de destruction massive, Paris, Éditions du Rocher et Le Serpent à plumes, 2005. 
tion particulière, mais bien des armées de tous les États, y compris des forces de maintien de la paix.

Les guerres tuent et mutilent sans distinction de sexe. Cependant, les femmes subissent d'autres exactions, exacerbées par les situations de conflit: les violences sexuelles. Que les soldats violent partout où ils passent n'est pas une nouveauté, c'est une tradition séculaire, visant à humilier et à terroriser les populations civiles ${ }^{49}$. Pour le « réconfort» des soldats, sont mis en place dans les pays sous occupation militaire des «camps de repos et de loisir». Des centaines de milliers de femmes et de jeunes filles tombent sous la coupe des réseaux de proxénètes qui, avec la bénédiction des gouvernements et de ses armées, tirent un juteux profit de la misère et du désordre.

Les crimes sexuels de masse, érigés en système par la prostitution militaire et la traite qui l'accompagne, sont en quelque sorte des invariants des guerres contemporaines. Les femmes et les fillettes y sont sexuellement et ethniquement infériorisées, tout en étant instrumentalisées et marchandisées afin de garantir le pouvoir, la supériorité et le plaisir masculins ainsi que le « déshonneur» des mâles nationaux subissant l'occupation. Par la suite, ces femmes et ces fillettes deviennent, via le système proxénète internationalisé, qui profite des dérèglementations néolibérales et qui s'appuie sur des politiques gouvernementales favorables aux industries du sexe, y compris au tourisme prostitueur, la source des rentes les plus profitables de l'économie mondialisée.

Fin du texte

49 Cela a été passé sous silence jusqu'à ces dernières années, mais depuis le Traité de Rome (1998), le viol de guerre est considéré comme un crime contre l'humanité. 